\title{
ÁGATA: um chatbot para difusão de práticas para Educação Ambiental
}

\author{
Bruno Rocha Gomes \\ brunogomess7@gmail.com \\ Universidade Estadual do Maranhão \\ São Luís, Brasil \\ Ivan de Jesus Pereira Pinto \\ navi1921@gmail.com \\ TeleMídia, PUC-Rio \\ Rio de Janeiro, Brasil
}

\author{
Antonio Fernando Lavareda Jacob Jr \\ antoniojunior@professor.uema.br \\ Universidade Estadual do Maranhão \\ São Luís, Brasil \\ Sérgio Colcher \\ colcher@inf.puc-rio.br \\ TeleMídia, PUC-Rio \\ Rio de Janeiro, Brasil
}

\begin{abstract}
Instant messaging apps have billions of monthly active users, which made this environment a good medium for purposes other than communication, such as education. In this context, one of the most appropriate technologies to encourage learning in these applications is chatbots. They consist of conversational applications that make use of artificial intelligence and natural language processing to interact with the user in a similar way to a human. In this scenario, this article presents the AGATE a chatbot developed for the Environmental Management Office (AGA / UEMA) in order to disseminate knowledge on environmental education, focusing on the problems of water and energy waste. The chatbot was developed for Telegram through the pyTelegramBotAPI implementation. In addition, conversation flows were created using DialogFlow. A multidisciplinary team composed of 14 people participated in the evaluation of the project, with a greater participation of professionals in the field of Biology. The initial results focused on the user experience were satisfactory.
\end{abstract}

\section{KEYWORDS}

Chatbots, digital education, environmental education, artificial intelligence, natural language processing

\section{INTRODUÇÃO}

A Educação Ambiental no Brasil é um tema discutido desde meados da década de 1960 [9]. Apesar de ter-se iniciado há algumas décadas, essa discussão se estende até os dias de hoje, havendo inclusive, produções recentes a respeito dos indicadores de monitoramento e avaliação de políticas públicas voltadas à educação ambiental [10]. Apesar desses esforços, o Brasil sofre com diferentes impactos ambientais frequentemente, sendo aqui destacado, o desperdício de água $^{1}$ e energia ${ }^{2}$, que tem crescido nos últimos anos. Dessa forma, a

\footnotetext{
${ }^{1}$ https://g1.globo.com/economia/noticia/2020/06/04/desperdicio-de-agua-aumentapelo-terceiro-ano-seguido-no-brasil-prejuizo-chega-a-r-12-bilhoes-apontaestudo.ghtml

${ }^{2}$ http://www.abesco.com.br/novidade/desperdicio-de-energia-atinge-r-617-bi-emtres-anos/

In: XIX Workshop de Ferramentas e Aplicações (WFA 2020), São Luís, Brasil. Anais Estendidos do Simpósio Brasileiro de Sistemas Multimídia e Web (WebMedia). Porto Alegre: Sociedade Brasileira de Computação, 2020.

(c) 2020 SBC - Sociedade Brasileira de Computação.

ISSN 2596-1683
}

necessidade de difundir a Educação Ambiental abriu possibilidades para que esta se apoiasse em meios tecnológicos, garantindo maior disponibilidade para o seu aprendizado.

Com o avanço tecnológico, uma nova modalidade de ensino surgiu, o Mobile Learning. Essa técnica, reconhecida pela UNESCO, consiste no uso de dispositivos móveis, tais como laptop, celular ou tablet, como instrumento de aprendizado [7]. Nesse cenário, destaca-se o uso de chatbots, cujo objetivo é simular uma conversa humana a partir da troca de mensagens automatizadas, por meio do uso de inteligência artificial e processamento de linguagem natural [11]. Tal tecnologia, é capaz de assumir diferentes comportamentos, tornando o processo de aprendizagem mais prazeroso e motivador [5]. Além disso, os chatbots podem ser integrados aos aplicativos de mensagem instantânea, tais como WhatsApp, Telegram e Facebook Messenger, além de websites e outras plataformas [1].

Levando em conta o uso de chatbots para educação, este trabalho visa apresentar a ÁGATA, um robô de conversação desenvolvido em parceria com a Assessoria de Gestão Ambiental (AGA/UEMA). A $\mathrm{AGA}^{3}$, cuja sigla inspirou a escolha do nome do chatbot, tem por objetivo desenvolver um Sistema de Gestão Ambiental (SGA) em todos os segmentos da sua atuação, resolvendo problemas socioambientais e desenvolvendo a consciência ecológica. A ÁGATA foi construída com o objetivo de promover a Educação Ambiental, através de dicas e curiosidades relacionadas a hábitos sustentáveis, no intuito de conter o desperdício de água e energia no Brasil. O chatbot foi avaliado a partir de um questionário composto por afirmações referentes às experiências dos usuários com a ÁGATA, sendo este respondido através da escala Likert de cinco níveis.

O restante deste artigo é composto da seguinte forma: na seção 2 será apresentado um referencial teórico a respeito dos chatbots; na seção 3 está contida a metodologia abordada no projeto; a seção 4 irá apresentar detalhes do desenvolvimento da ÁGATA; a seção 5 mostrará as avaliações da aplicação desenvolvida; e, por fim, a seção 6 contará com as considerações finais e trabalhos futuros.

\section{REFERENCIAL TEÓRICO}

Em 1950, foi publicado um artigo que seria um dos pioneiros na área de Inteligência Artificial (IA). "Computing Machinery and Intelligence", por Alan Turing, trouxe à tona o questionamento sobre uma máquina ser capaz de pensar. Com isso, Turing propôs o "Teste

\footnotetext{
${ }^{3}$ https://www.aga.uema.br
} 
de Turing" (também chamado de "Jogo da Imitação"), que consiste em um entrevistador tentar distinguir se está conversando com um ser humano ou uma máquina. Dessa forma, se o entrevistador não for capaz de fazê-lo, a máquina é considerada inteligente [3].

Motivados pelo desafio proposto por Turing, vários pesquisadores começaram a desenvolver sistemas que utilizavam o Processamento de Linguagem Natural (PLN), sendo o primeiro deles o software ELIZA, criado por Joseph Weizenbaum, em 1966. O sistema tinha como objetivo simular a conversa entre um psicólogo e seu paciente através da técnica de casamento de padrões [13]. Anos mais tarde, surgiu A.L.I.C.E (Artificial Linguistic Internet Computer Entity), um novo robô de conversação, desenvolvido por Richard S. Wallace, que inovou por utilizar no seu desenvolvimento o AIML, uma linguagem de marcação para inteligência artificial baseada no XML [12]. Diante disso, o surgimento desses sistemas computacionais motivou a criação de competições que premiam as aplicações mais inteligentes, como é o caso do Loebner Prize e o ChatterBox Challenge [3].

O termo chatterbots (ou chatbots - robôs de conversação) foi utilizado pela primeira vez por Michael L. Mauldin, em 1994, para denominar esse tipo de sistema que estava sendo desenvolvido até então [6]. Também conhecidos como agentes conversacionais, ou apenas bot, essas aplicações consistem em robôs virtuais capazes de se comunicar com um humano, compreendendo o contexto do que é dito e respondendo conforme o entendimento, de forma que simule a linguagem natural de maneira automatizada [2]. Os chatbots são divididos em dois tipos: baseado em regras e baseado em IA. O primeiro, faz uso de fluxos de conversa pré-definidos, com perguntas e expressões cadastradas previamente em uma base de dados. Já o baseado em IA, o qual a ÁGATA se encaixa, utiliza o processamento de linguagem natural para o seu treinamento a fim de identificar contextos de uma conversa e responder corretamente às intenções do usuário [1].

Os chatbots possuem a grande vantagem de ter disponibilidade durante os 7 dias da semana, além de funcionarem em múltiplos canais de comunicação de forma simultânea para vários usuários, o que permite automatizar processos de diferentes naturezas [1] Com isso, essas aplicações podem ser desenvolvidas para diversas finalidades, como por exemplo, educacional, entretenimento, perguntas mais frequentes (do inglês, Frequent Asked Questions - FAQ), comercial e propósitos gerais [4].

Na literatura técnica, é possível encontrar vários chatbots desenvolvidos com diferentes propósitos. O Robô Ed [8], um dos poucos voltados à Educação Ambiental, é um chatbot criado em 2004 para o CONPET, um Programa do Governo Federal vinculado ao Ministério de Minas e Energia e com apoio técnico, administrativo e financeiro da Petrobras. Trata-se de um robô de conversação cujo objetivo é discutir sobre a preservação dos recursos naturais e energéticos, abordando temas como a conservação das energias não renováveis, preservação do meio ambiente e ecologia em geral. O PipaBot [1], por sua vez, foi construído para o Projeto Infância e Poluentes Ambientais (PIPA UFRJ), com intuito de acompanhar mães e bebês, avaliando os efeitos de poluentes ambientais no período de gestação até o início da infância. Este foi desenvolvido para o Facebook Messenger e para o website do PIPA-UFRJ, a partir do Botpress framework. No contexto de chatbots para educação, também é possível encontrar trabalhos correlatos. Donuts [7] é um bot desenvolvido com o propósito de facilitar o ensino da disciplina "Construção de Algoritmos"nos cursos de Computação. Desenvolvido a partir da Bot API da plataforma Telegram e na linguagem Python, o Donuts funciona no formato de quiz com elementos de gamificação, tais como pontuações, ranking e sistema de punições e recompensas. Por fim, o English Coach [11] é um robô de conversação projetado para auxiliar estudantes do ensino médio no aprendizado da língua inglesa, oferecendo exercícios de gramática e pronúncia do idioma. Foi desenvolvido na linguagem fava para a plataforma Telegram, utilizando o banco de dados PostgreSQL e sendo hospedado na plataforma Google Cloud.

\section{METODOLOGIA}

A ÁGATA foi desenvolvida seguindo a metodologia ágil Scrum ${ }^{4}$. Nesta, o projeto é divido em ciclos, chamados sprints, que traçam pequenas tarefas ou funcionalidades que devem ser implementadas na aplicação. Dessa forma, havia reuniões semanais para a coleta de feedbacks do que foi feito e para que novos sprints fossem definidos.

Sabendo que o projeto seria realizado em parceria com a AGA, logo concretizou-se a ideia de utilizar o chatbot para promover conhecimentos relacionados à Educação Ambiental. Além disso, ao pesquisar trabalhos correlatos foi possível perceber que não havia muitos robôs de conversação voltados a esse gênero na literatura técnica. Dessa forma, foram realizadas reuniões com a Assessoria de Gestão Ambiental a fim de definir qual problemática da educação ambiental seria trabalhada e a qual plataforma a aplicação seria integrada.

(1) Foi decidido que o tema principal abordado pela ÁGATA seria referente à preservação de água e energia no país, visto que o Brasil tem sofrido com o aumento do desperdício desses dois recursos. Sendo assim, a AGA disponibilizou um de seus materiais ${ }^{5}$ contendo ensinamentos e curiosidades a respeito da problemática, a fim de que fossem elaboradas frases para a aplicação.

(2) Para definir a plataforma que iria integrar a ÁGATA, foi considerada a disponibilidade e preços das APIs de diferentes aplicativos de mensagens instantâneas. Nesse contexto, foi escolhido o Telegram, uma vez que sua API é pública e a plataforma fornece um apoio ao desenvolvimento de chatbots, incentivando APIs da comunidade e disponibilizando uma documentação completa para seu uso. Além disso, trabalhos correlatos já evidenciaram obter sucesso com o uso dessa plataforma [7] [11].

\section{4 ÁGATA}

Nas subseções seguintes, será apresentada a arquitetura, o fluxo de conversa e os detalhes da implementação da ÁGATA.

\subsection{Arquitetura}

A arquitetura do chatbot é baseada no modelo cliente-servidor, conforme ilustra a Figura 1. O lado cliente, consiste na interface por onde o usuário interage com a aplicação, ou seja, a própria interface

\footnotetext{
${ }^{4} \mathrm{https} / /$ www.desenvolvimentoagil.com.br/scrum/

${ }^{5}$ https://www.aga.uema.br/wp-content/uploads/2018/09/CARTILHA-2.1-CAPANOVA.pdf
} 
do Telegram. Já o lado servidor, é composto por duas partes: o servidor do Telegram e o servidor da ÁGATA.

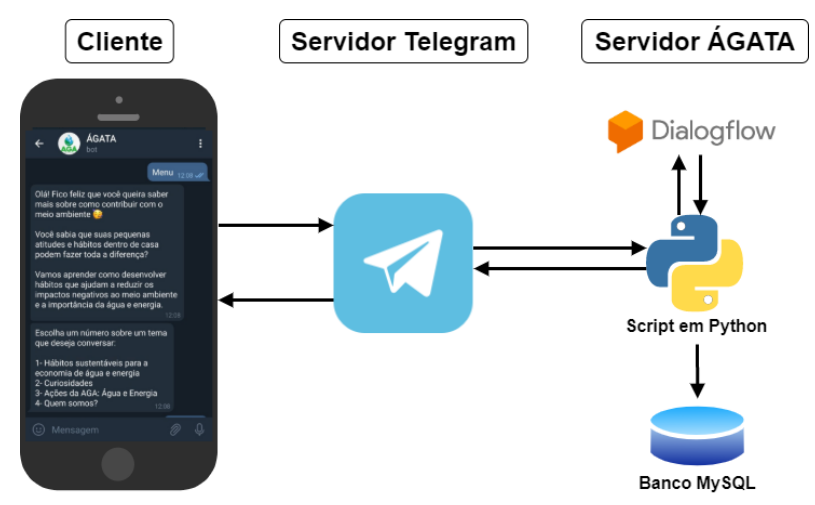

Figura 1: Arquitetura da ÁGATA

O servidor do Telegram é responsável por intermediar o lado cliente com o servidor da ÁGATA. Assim, todas as mensagens enviadas para o bot pelo usuário, serão primeiramente encaminhadas para este componente da arquitetura, que por sua vez, é encarregado de repassá-las para o servidor do chatbot.

O servidor da ÁGATA consiste em um script escrito na linguagem de programação Python, sendo responsável por fazer o tratamento das mensagens enviadas pelo usuário. Assim que o servidor do Telegram repassa as mensagens obtidas no lado cliente, tal script se comunica com a plataforma Dialogflow, responsável por armazenar todo o fluxo de conversa e base de conhecimento do bot, a fim de obter a resposta mais adequada à interação do usuário. Dessa forma, uma vez que a resposta é selecionada, o script salva as informações da conversa em um banco de dados $M y S Q L$ e por fim, encaminha a mensagem de volta para o servidor do Telegram, que a repassa ao usuário.

\subsection{Fluxo de conversa}

A construção do fluxo de conversa da ÁGATA se deu em conjunto com a AGA UEMA. Com base no material fornecido por esta, foi organizado em um mind map todas as possíveis interações com o chatbot, que em seguida, foram avaliadas pela Assessoria de Gestão Ambiental. O diálogo com a ÁGATA ocorre através de menus numéricos ou interações e perguntas soltas. Dessa forma, o usuário pode chegar à informação desejada tanto através do menu quanto através de questionamentos.

Ao interagir com ÁGATA pela primeira vez, o usuário irá enviar o comando padrão do Telegram "/start", disparando assim, o menu principal do bot. Tal recurso também pode ser acessado digitando "menu"no chat ou enviando uma mensagem de saudação. Conforme mostra na Figura 2, as opções fornecidas pela ÁGATA vão desde dicas e curiosidades sobre educação ambiental, as quais direcionam o usuário para um fluxo de mensagens do tema, até informações sobre a própria AGA. Mais detalhes sobre as possíveis interações com a ÁGATA podem ser encontrados no vídeo de demonstração ${ }^{6}$ do chatbot.

\footnotetext{
$\overline{{ }^{6} \text { https://youtu.be/RsuvY444D1w }}$
}

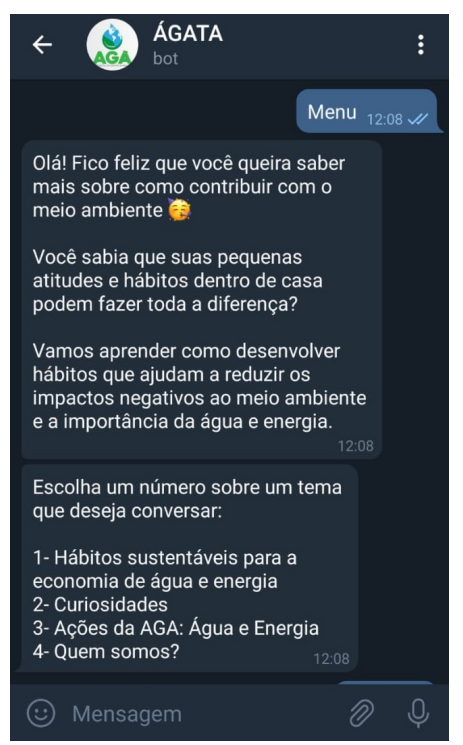

Figura 2: Menu principal da ÁGATA

A implementação desse fluxo de mensagens no bot se deu através da ferramenta Dialogflow, na sua versão Essentials. Essa plataforma do Google, é voltada para a construção de agentes conversacionais com base no processamento de linguagem natural e aprendizado de máquina, podendo integrá-los a inúmeros aplicativos de mensagens, websites, dispositivos e serviços ${ }^{7}$.

O Dialogflow permite que sejam criadas intents, ou intenções do usuário, para o agente conversacional. Cada intent permite adicionar frases de treinamento e suas possíveis respostas. Com isso, após o treinamento do chatbot, a partir das técnicas de PLN e aprendizado de máquina disponíveis no próprio Dialogflow, este é capaz de identificar cada intenção através das mensagens de entrada do usuário, retornando assim, uma das mensagens de saída definida na intent em questão. Entretanto, caso uma frase não seja identificada, será ativada uma fallback intent, informando que a mensagem não foi entendida e, no caso da ÁGATA, é sugerido que o usuário envie a palavra "menu"para exibir as opções do bot.

Cada intent permite, ainda, adicionar contextos de conversa. Eles são responsáveis pelo chatbot fornecer respostas distintas para frases iguais, quando estas se encontram em diferentes contextos. $\mathrm{Na}$ ÁGATA, tal recurso foi necessário, pois como existem vários menus numéricos no fluxo de conversa, um mesmo número poderá resultar em respostas diferentes, dependendo do contexto atual, ou seja, de qual menu o usuário está se referindo.

\subsection{Detalhes de implementação}

Para o desenvolvimento da ÁGATA, foi elaborado um script em Python, na sua versão 3.8.5. Nele, utilizou-se a pyTelegramBotAPI ${ }^{8}$, uma implementação escrita na mesma linguagem e que faz uso da Bot API ${ }^{9}$ do Telegram, bem como da framework Flask. Além disso, para que a ÁGATA funcionasse 24 horas por dia, a aplicação

\footnotetext{
${ }_{7 \text { https://cloud.google.com/dialogflow/docs/ }}$

${ }^{8}$ https://github.com/eternnoir/pyTelegramBotAPI

${ }^{9}$ https://core.telegram.org/bots/api
} 
foi hospedada na plataforma em nuvem Heroku. Sendo assim, foi utilizado o formato webhook para notificar o servidor sempre que um novo evento ocorrer, ou seja, quando uma nova interação com a ÁGATA surgir.

O script principal também conta com a biblioteca PyMySQL, do Python. Ela é responsável por permitir a conexão com o banco de dados $M y S Q L$ da aplicação. Este por sua vez, consiste em um banco simples composto por duas tabelas: TbUsuario e TbConversa. A primeira, contém informações como nome, sobrenome e um identificador de usuário do Telegram. Já a segunda, contém as mensagens de entrada e de saída, data da interação e uma chave estrangeira para o usuário. Tais informações são importantes para identificar os interesses de cada usuário no tema em questão, permitindo traçar possíveis melhorias nos fluxos de conversa da ÁGATA.

O backend da aplicação é formado, ainda, por dois scripts. Um deles é responsável apenas por realizar as operações do banco de dados, tais como buscas na tabela TbUsuario e inserção de novos usuários e registros de conversas. O outro, por sua vez, é responsável por consumir a API do Dialogflow ${ }^{10}$ a fim de consultar o agente conversacional criado na plataforma, permitindo assim, detectar a intenção do usuário a partir da mensagem de entrada e retornar a resposta mais adequada para o script principal.

O código fonte da ÁGATA está disponível no Github ${ }^{11}$, possuindo licença copyleft através da General Public License (GPL).

\section{AVALIAÇÃO DA ÁGATA}

Para a etapa de avaliação da ÁGATA, reuniu-se uma equipe multidisciplinar com maior participação de profissionais da área de Biologia, totalizando 14 pessoas, sendo 11 destas, participantes da AGA e o restante composto por alunos de graduação do curso de Ciências Biológicas da UEMA. Foi solicitado a todos que utilizassem o chatbot, explorando todas as opções oferecidas no seu menu principal, além das interações soltas. Por fim, cada um dos avaliadores foi convidado a responder um questionário relacionado às suas experiências com a ÁGATA. O questionário, mostrado na Tabela 1, é composto por 12 afirmações, devendo ser respondido através da escala Likert de cinco níveis (Concordo totalmente, Concordo parcialmente, Neutro, Discordo parcialmente e Discordo totalmente).

Na subseção seguinte, será apresentada uma análise quantitativa das respostas obtidas a partir do questionário, a fim de avaliar a experiência proporcionada pela ÁGATA.

\subsection{Resultados obtidos}

Conforme mostrado na Tabela 2, a ÁGATA recebeu, no geral, avaliações positivas. Das doze afirmativas contidas no questionário, oito obtiveram respostas unicamente no quadrante positivo, ou seja, foram respondidas como "concordo totalmente"ou "concordo parcialmente". As afirmações A1 e A8 tiveram a maior taxa de respostas "concordo totalmente", representando $100 \%$ dos avaliadores. A5 e A7, por outro lado, foram as únicas avaliadas no quadrante negativo, representando 7,1\% das respostas em cada. Por fim, apenas as afirmativas A4 e A6 representaram a concordância "neutra"do grupo de avaliadores (cerca de 14,3\% das respostas em cada).

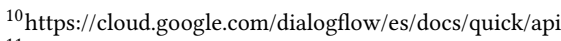

${ }^{11}$ https://github.com/lincprog/agata
}

Tabela 1: Afirmações contidas no questionário

\begin{tabular}{|l|l|}
\hline A1 & $\begin{array}{l}\text { No início, fiquei interessado pelo tema proposto pela } \\
\text { ÁGATA }\end{array}$ \\
\hline A2 & $\begin{array}{l}\text { O conteúdo abordado pela ÁGATA é compatível com os } \\
\text { ensinamentos relacionados à educação ambiental }\end{array}$ \\
\hline A3 & $\begin{array}{l}\text { A ÁGATA fornece respostas pertinentes aos meus ques- } \\
\text { tionamentos }\end{array}$ \\
\hline A4 & $\begin{array}{l}\text { O funcionamento da ÁGATA está adequado ao meu jeito } \\
\text { de aprender }\end{array}$ \\
\hline A5 & A ÁGATA é de fácil entendimento e uso \\
\hline A6 & $\begin{array}{l}\text { A ÁGATA me incentivou a aprender mais sobre hábitos } \\
\text { sustentáveis da educação ambiental }\end{array}$ \\
\hline A7 & $\begin{array}{l}\text { Eu pretendo continuar utilizando a ÁGATA para apren- } \\
\text { der mais sobre hábitos sustentáveis }\end{array}$ \\
\hline A8 & $\begin{array}{l}\text { Gostaria de utilizar a ÁGATA para aprender sobre outros } \\
\text { temas da educação ambiental }\end{array}$ \\
\hline A9 & $\begin{array}{l}\text { A ÁGATA responde rapidamente aos meus questiona- } \\
\text { mentos }\end{array}$ \\
\hline A10 & $\begin{array}{l}\text { Eu consigo obter a informação desejada com poucas } \\
\text { interações }\end{array}$ \\
\hline A11 & Eu gostei de utilizar a ÁGATA \\
\hline A12 & $\begin{array}{l}\text { Usar a ÁGATA pode aumentar meu desempenho no pro- } \\
\text { cesso de ensino e aprendizado da educação ambiental }\end{array}$ \\
\hline
\end{tabular}

É possível considerar, com base nas afirmações com maior taxa de aceitação (A5 e A8), que o tema da Educação Ambiental foi muito bem aceito pelos avaliadores. Deve-se levar em conta, que a maioria do grupo participante da avaliação tem domínio na área, o que ajuda a explicar o interesse pelo assunto abordado pela ÁGATA. Além disso, por haver poucos chatbots com esse propósito, a experiência dos avaliadores tendeu a ser inovadora.

Afirmativas como A4 e A6, que obtiveram respostas neutras, e A7, que obteve $7,1 \%$ das respostas como "discordo parcialmente", sugerem que novos recursos possam ser aplicados à ÁGATA para despertar ainda mais o interesse dos usuários em utilizar o chatbot para a educação ambiental, mesmo obtendo mais de $80 \%$ de aprovação nesse quesito. Nesse mesmo contexto, a afirmação A5 chamou atenção por ser a única contendo respostas do tipo "discordo totalmente", representando apenas 7,1\% do total. Dessa forma, tal resultado sugere que a ÁGATA deve fornecer mais explicações sobre seu uso, assim como levanta a possibilidade de explorar mais elementos intuitivos, tais como os botões de navegação, disponíveis no Telegram, ou mensagens por voz, disponível no Dialogflow.

Em geral, a ÁGATA obteve resultados satisfatórios na avaliação. O chatbot cumpriu o seu papel ao passar ensinamentos relacionados à educação ambiental, tendo seu conteúdo compatível com o tema proposto, conforme aponta os resultados em A2 e A3. Além disso, essa etapa do projeto levantou possibilidades de trabalhos futuros.

\section{CONSIDERAÇÕES FINAIS}

Este artigo apresentou a ÁGATA, um chatbot construído em parceria com a AGA UEMA, com o objetivo de promover ensinamentos da área de Educação Ambiental, abordando a problemática do desperdício de água e energia no Brasil. 
Tabela 2: Respostas obtidas na avaliação

\begin{tabular}{|c|c|c|c|c|c|c|c|c|c|c|c|c|}
\hline & A1 & A2 & A3 & A4 & A5 & A6 & A7 & A8 & A9 & A10 & A11 & A12 \\
\hline Concordo Totalmente & 14 & 13 & 13 & 10 & 12 & 12 & 11 & 14 & 13 & 9 & 13 & 10 \\
\hline Concordo Parcialmente & - & 1 & 1 & 2 & 1 & - & 2 & - & 1 & 5 & 1 & 4 \\
\hline Neutro & - & - & - & 2 & - & 2 & - & - & - & - & - & - \\
\hline Discordo Parcialmente & - & - & - & - & - & - & 1 & - & - & - & - & - \\
\hline Discordo Totalmente & - & - & - & - & 1 & - & - & - & - & - & - & - \\
\hline
\end{tabular}

Através da ÁGATA, é possível aprender dicas e curiosidades sobre hábitos sustentáveis para combater o tema em questão, além de conhecer mais sobre os objetivos e ações promovidas pela AGA. Com base nas pesquisas feitas na literatura técnica foi possível perceber ainda, que existem poucos chatbots voltados para o ensino da Educação Ambiental, sendo este um trabalho do gênero totalmente gratuito e disponível em uma plataforma de conversação atual, o Telegram. Além disso, a aplicação foi avaliada por uma equipe multidisciplinar (com maior participação de profissionais da área de Biologia), através de um questionário relacionado às experiências dos usuários com o chatbot. Dessa forma, obteve-se resultados satisfatórios nesta primeira versão.

Embora tenha cumprido bem o seu propósito, a ÁGATA ainda apresenta certas limitações, tais como a problemática abordada e o fato de estar disponível apenas na plataforma Telegram. Além disso, a avaliação do trabalho foi comprometida por conta da pandemia do COVID-19, limitando a quantidade de participantes na pesquisa Sendo assim, é importante considerar alguns trabalhos futuros:

(1) Conforme visto na avaliação da ÁGATA, um dos pontos de maior aceitação é a possibilidade de utilizar o chatbot para aprender sobre outros temas da Educação Ambiental. Por isso, planeja-se criar novos fluxos de conversa abordando outras problemáticas que vão além do desperdício de água e energia.

(2) Planeja-se também, fazer a integração da ÁGATA a outros aplicativos de mensagem instantânea, tais como Facebook Messenger e WhatsApp. Para a atual versão do Telegram, a ideia é explorar mais recursos oferecidos pela plataforma, como os botões de navegação, por exemplo.

(3) Outro recurso que será explorado é o reconhecimento por voz, oferecido pelo Dialogflow. Tal propriedade irá garantir à ÁGATA maior acessibilidade ao seu conteúdo.

(4) Por fim, pretende-se ampliar a quantidade de participantes na avaliação da ÁGATA, incluindo mais usuários leigos e que não tiveram contato com o desenvolvimento do projeto. Assim, será possível realizar uma avaliação de desempenho comparando os resultados obtidos entre os entendedores do assunto e aqueles sem conhecimento prévio do tema.

\section{REFERÊNCIAS}

[1] Lucas S de Paula, Taisa G Gonçalves, Thatiana VRB Fernandes, and Guilherme $\mathrm{H}$ Travassos. 2019. PipaBot: um canal de comunicação para o PIPA UFRJ. In Anais Estendidos do XXV Simpósio Brasileiro de Sistemas Multimídia e Web. SBC, 103 107.

[2] Marília Guimarães dos Santos and Antonio Carlos dos Santos Souza. [n.d.]. WALO: Ferramenta para Gerenciamento de Mídias Sociais. ([n. d.]).
[3] Antonio Fernando Lavareda Jacob Junior. 2008. Buti: um companheiro virtual baseado em computação afetiva para auxiliar na manutenção da saúde cardiovascular. Master's thesis. Universidade Federal de Pernambuco.

[4] Matheus Canali Fossatti, Roberto dos Santos Rabello, and Ana Carolina Bertoletti de Marchi. 2011. AGEbot: um chatterbot em AIML voltado para responder questões sobre Epilepsia. In Anais do XXXI Congresso da Sociedade Brasileira da Computação. sn.

[5] Antonio FL Jacob Jr, Diego L da C Silva, Áveles de S Oliveira Filho, Flávia de A Barros, Carlos RL Francês, and João CWA Costa. [n.d.]. Elicitação do Conhecimento por meio de Ontologias para Criação de um Companheiro Virtual de Aprendizado para Promoção da Saúde Cardiovascular na Infância e Pré-Adolescência. ([n. d.]).

[6] Michael L Mauldin. 1994. Chatterbots, tinymuds, and the turing test: Entering the loebner prize competition. In $A A A I$, Vol. 94. 16-21.

[7] Ceres GB Morais, Alexsandra F Gomes, Jéssica N de F Leite, K de A Kléber, and Thiago J Barbalho. 2017. Donuts: um bot como instrumento facilitador do processo de ensino-aprendizagem na disciplina "Construção de Algoritmos". Revista Eletrônica Argentina-Brasil de Tecnologias da Informação e da Comunicação 1, 7 (2017).

[8] Marilís Pereira Lima Moura, Maria Elisabeth dos Santos Rodrigues, Rafaele Paz Comin, Washington de Jesus Sant, et al. 2019. Prospecção de Tecnologias em Chatbots. Cadernos de Prospecção 12, 3 (2019), 550.

[9] Daniele Lopes Oliveira. 2020. Educação Ambiental no Brasil: Estudo de Caso Escolas Municipais da Zona Urbana e Rural de Jaraguá-Goiás. Humanidades e Tecnologia em Revista (FINOM) 1, 23 (2020), 192-212.

[10] Maria Henriqueta Andrade Raymundo, Evandro Albiach Branco, Semiramis Biasoli, Marcos Sorrentino, and Renata Rozendo Maranhão. 2019. Caderno de Indicadores de avaliação e monitoramento de políticas públicas de Educação Ambiental: processo de construção participativa e fichas metodológicas. CEP 12 (2019), 010.

[11] Saulo Henrique Cabral Silva, Marcos Vinícius de Souza Toledo, Luísa Calegari de Barros Cizilio, and Iago Izidório Lacerda. 2020. O uso de chatbot no auxílio de estudantes do ensino médio para o aprendizado em inglês/The use of chatbot in the help of high school students for english learning. Brazilian fournal of Development 6, 1 (2020), 2933-2944.

[12] Richard S Wallace. 2009. The anatomy of ALICE. In Parsing the Turing Test. Springer, 181-210.

[13] Joseph Weizenbaum. 1966. ELIZA-a computer program for the study of natural language communication between man and machine. Commun. ACM 9, 1 (1966), $36-45$. 